\title{
Effects of Microgravity on Action Potential Wave Propagation in Rat Transmural Ventricle Tissue
}

\author{
Xiangyun Bai ${ }^{1}$, Kuanquan Wang ${ }^{1}$, Qince $\mathrm{Li}^{1}$, Cunjin Luo $^{2}$, Yacong Li ${ }^{1}$, Henggui Zhang ${ }^{1,2,3,4}$, \\ ${ }^{1}$ School of Computer Science and Technology, Harbin Institute of Technology, Harbin, Heilongjiang, \\ China \\ ${ }^{2}$ Key Laboratory of Medical Electrophysiology, Ministry of Education, Collaborative Innovation \\ Center for Prevention and Treatment of Cardiovascular Disease/Institute of Cardiovascular Research, \\ Southwest Medical University, Luzhou, China \\ ${ }^{3}$ Biological Physics Group, School of Physics and Astronomy, The University of Manchester, \\ Manchester, UK \\ ${ }^{4}$ Space Institute of Southern China, Shenzhen, China
}

\begin{abstract}
Experiments mimicking microgravity condition have multiple effects on cardiac electrophysiology. Therefore, based on experimental data of rats with 2-weeks (short term) and 4-weeks (long term) tail suspension, effect of microgravity on action potentials (APs) were simulated by decreasing $I_{C a L}$ and increasing $I_{N a K}$ based on rat endocardial and epicardial models. Additionally, a $1 D$ model was constructed by considering the increasing of connexin 43 under microgravity condition. Simulation results show that $I_{C a L}$ and $I_{N a K}$ changes have similar effect in reducing $A P D_{90}$ under short term condition, while the inhibition of $I_{C a L}$ is the main factor in reducing $A P D_{90}$ under long term condition. The simulated pseudo-ECG in both conditions showed a shortened $Q T$ interval and depressed of $S T$ phase and $T$ wave as experimental observations. Increased expression of connexin 43 in microgravity condition resulted in a mild increase in conduction velocity. Meanwhile, the vulnerable window in $1 D$ ventricle strand reduced in microgravity condition. Compared with short term microgravity condition, all these changes were more prominent in the long term microgravity condition. In conclusion, this study provides new insight into understanding of impaired cardiac functions in short and long term microgravity conditions during spaceflight.
\end{abstract}

\section{Introduction}

In condition of removing force of gravity in actual spaceflight or simulated microgravity (eg., rat tail suspension) causes back flow of blood from the lower part of the body into the upper part, affecting body organs such as brain and heart. Previous experiments have reported changes of membrane currents and the expression of connexion 43 in ventricular myocytes in the condition of rat tail suspension, including currents of $\mathrm{L}$ type $\mathrm{Ca}^{2+}$ current $\left(I_{\mathrm{CaL}}\right)$ [1,2] and $\mathrm{Na}^{+}-\mathrm{K}^{+}$pump current $\left(I_{\mathrm{NaK}}\right)$ [3]. These changes which affect the heterogeneity of action potential duration (APD) of endocardial and epicardial myocytes could be pro-arrhythmic. It has been reported that the occurrence of cardiac arrhythmia of an astronaut during spaceflight resulted in an earlier termination of the space trip [4]. Therefore, it is important to identify the mechanism of arrhythmogenesis in microgravity condition and for further development of proper treatment.

In order to explore the above issues, biophysically detailed rat endocardial and epicardial models were developed based on experimental data of rat tail suspension [3] to examine the effect of microgravity on the activity of the heart. Using developed heterogeneous ventricular cell models, the main factor(s) affecting action potential (AP) wave generation in the short term and long term microgravity conditions were revealed. In addition, the propagation of AP waves in the 1-dimentional (1-D) ventricle strand was also investigated using the constructed 1-D ventricle strand model. This simulation results may provide a theoretical therapy target for further anti-arrhythmia researches under microgravity condition.

\section{Methods}

\subsection{Ventricular cell models and modeling methods}

The Pandit et al. models [5] of action potentials of rat endocaridal and epicardial cells were modified to mimick 
the effects of microgravity. Its impact on $I_{\mathrm{CaL}}$ was simulated by blocking of $I_{\mathrm{CaL}}$ based on experimental data [3]. Meanwhile, the increasing of $I_{\mathrm{NaK}}$ also incorporated because of the up-regulated expression of $\mathrm{Na}^{+}-\mathrm{K}^{+}$ATPase $\alpha 2$ sub-unit in ventricular myocytes in microgravity experiment of rat tail suspension [3]. Two conditions including short term ( 2 weeks) and long term (4 weeks) microgravity were defined to represent the effect of different length of microgravity on ventricular myocytes according to experimental data [3] summarized as follows (Table 1):

Table 1. Membrane currents changes in rat endocardial and epicardial ventricular myocytes in microgravity conditions.

\begin{tabular}{ccc}
\hline $\begin{array}{c}\text { weightlessness } \\
\text { (rat tail suspension) }\end{array}$ & $\downarrow \boldsymbol{I}_{\mathrm{CaL}}(\%)$ & $\uparrow \boldsymbol{I}_{\mathrm{NaCa}}(\%)$ \\
\hline Short term (2 weeks) & 9.1 & 58.5 \\
Long term (4 weeks) & 50.9 & 106.9 \\
\hline
\end{tabular}

The AP for ventricular myocytes represented as in previous study [5] which was solved by explicit Euler method in a time step of $0.0001 \mathrm{~ms}$. For inducing the AP waves, a series of supra-threshold stimuli with strength of $0.6 \mathrm{nA}$ were applied to the endocardial and epicardial myocytes for $5 \mathrm{~ms}$ at a frequency of $5 \mathrm{~Hz}$.

\subsection{1-D ventricle transmural strand model and numerical approach}

A model of 1D ventricle transmural strand was constructed, with the AP for ventricular cells in this strand was described as follows:

$$
\frac{\partial V}{\partial t}=-I_{\text {ion }}+\nabla \cdot(D \nabla V),
$$

Where $D$ is diffusion coefficient and equals to 0.0113 $\mathrm{mm}^{2} / \mathrm{s}$. It has been shown that the expression of connexin 43 mildly up-regulated in the short and long term microgravity conditions based on rat tail suspension data [3], increasing by $12 \%$ and $11 \%$ respectively. Therefore, we set the value of $D$ in the 1D strand increased by the same proportions as described above.

The length of the $1 \mathrm{D}$ ventricle strand is $3 \mathrm{~mm}$, including $2 \mathrm{~mm}$ of endocardial strand and $1 \mathrm{~mm}$ of epicardial strand. It was assumed to be equivalent to include 20 endocardial cells and 10 endocardial cells based on our simulation used a spatial resolution of $0.1 \mathrm{~mm}$ which is equal to the length of ventricular cells. Standard S1-S2 stimuli protocol was applied to activate the tissue and calculated vulnerable window of the 1D strand: S1 stimuli were applied for $5 \mathrm{~ms}$ to the first 3 endocardial cells to induce the excitation propagation in the tissue, and then S2 stimuli simultaneously applied to the $11^{\text {th }}$ cell and the following 5 connected cells.

\section{Results}

\subsection{Computer modeling short and long term microgravity effects on action potentials in ventricular cells}
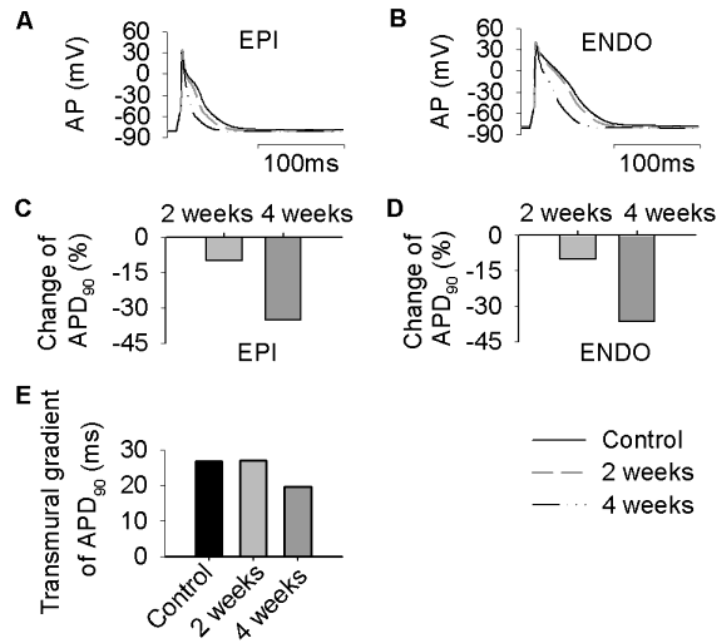

Fig1. Simulated action potentials in endocardial and epicardial ventricular cells in short and long term microgravity conditions.'

Figure 1 represents the effects of the short and long term microgravity on the APs in endocardial and epicardial myocytes. Simulation results show that the APD reduced in both endocardial and epicardial cells in microgravity conditions (figure $1 \mathrm{~A}$ and $1 \mathrm{~B}$ ). Short term microgravity decreased similar proportion of $\mathrm{APD}_{90}$ in endocardial and pericardial myocytes, decreasing by approximately $10 \%$ (figure $1 \mathrm{C}$ and 1D). While the reduction of $\mathrm{APD}_{90}$ is more prominent in the long term microgravity condition, reducing by $34.9 \%$ in endocardial myocyte and $36.5 \%$ in epicardial myocyte (figure $1 \mathrm{C}$ and 1D). This heterogeneous APD reduction resulted in a reduced transmural dispersion of $\mathrm{APD}_{90}$ by $7.1 \mathrm{~ms}$ in the long term microgravity condition (figure 1E).

\subsection{Effect of microgravity induced changes in ion channel alone on action potential duration in ventricular myocytes}

A reduction of $I_{\mathrm{CaL}}$ and an augment of $I_{\mathrm{NaK}}$ have been observed experimentally in microgravity condition. However, the relative contributions of these two changed currents in microgravity condition are unclear. Therefore, the functional effect of microgravity induced changes in ionic currents on $\mathrm{APD}_{90}$ was computed separately (figure 2). Simulation results show that reduction of $I_{\mathrm{CaL}}$ played a dominant role in reducing $\mathrm{APD}_{90}$ in both epicardial and 
endocardial cells in the long term microgravity condition, as well as in epicardial cell in the short term microgravity condition. While the two altered currents have similar effect on $\mathrm{APD}_{90}$ in endocardial cell in the short term microgravity condition.

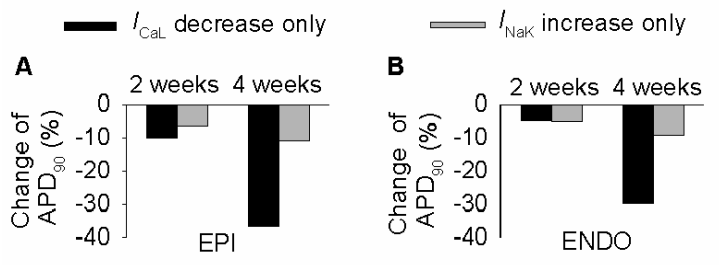

Fig2. The effect of microgravity induced changes in ion channel alone on $\mathrm{APD}_{90}$ in endocardial and epicardial myocytes.

\subsection{Simulated excitation wave propagation across the $1 \mathrm{D}$ ventricle tissue in short and long term microgravity conditions}

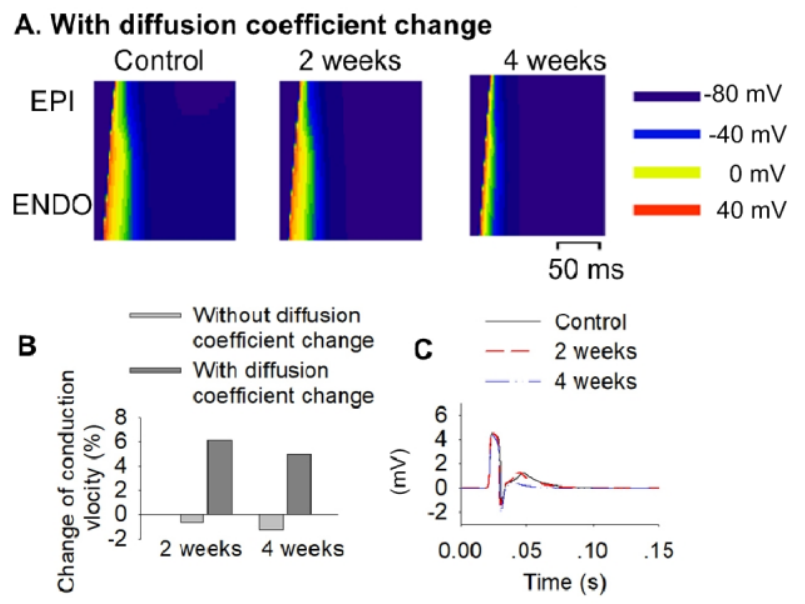

Fig 3. Excitation propagation across the 1D ventricular tissue without and with diffusion coefficient changes in control, short and long term microgravity conditions.

Experimental observation shows that the expression of connexin 43 up-regulated in microgravity condition [3]. Therefore, we simulated AP wave propagation in the 1D strand with an increased diffusion coefficient $(D)$. The AP induced from the beginning of endocardial myocytes and propagated to the direction of epicardial myocytes in the strand (figure 3A). It is shown that repolarization of the AP accelerated from the short term to the long term microgravity condition (figure 3A). Microgravity increased $D$ resulted in conduction velocity from negligible reduction turned to mild increase, increasing by $6 \%$ and $5 \%$ respectively in the short and long term microgravity conditions (figure 3B). Microgravity induced ionic currents changes decreased APD, this APD reduction is strongly correlated with the QRS and T wave in electrocardiogram (ECG). Therefore, a pseudo-ECG was computed in control and microgravity conditions (figure 3C). Simulation results show that the long term microgravity resulted in a reduction of QT interval as well as a depression of ST phase and the peak of T wave, which are consistent with experimental observation [3]. Meanwhile, it is shown that the peak of $\mathrm{T}$ wave and ST phase depressed obviously from the short to long term microgravity conditions.

\subsection{Simulated temporal vulnerability of the 1D ventricle strand to unidirectional block in the short and long term microgravity conditions}

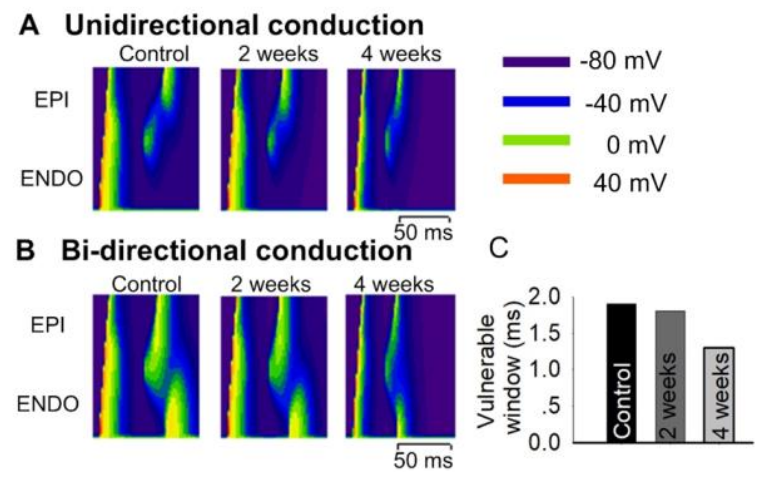

Figure 4. Simulated temporal vulnerabilities of the tissue to unidirectional conduction block in response to a premature stimulus in control and microgravity conditions.

Further studies were carried out to identify how the short and long term microgravity changed the susceptibility of the 1D tissue to arrhythmogenesis. Using the developed 1D tissue, a test stimulus applied to the refractory tail of previous excitation wave to quantitatively compute the temporal vulnerability of the 1D strand to unidirectional block under control, the short and long term microgravity conditions. With the time interval between $\mathrm{S} 1$ and $\mathrm{S} 2$ stimuli $(\Delta \mathrm{t})$ equal to $81.5 \mathrm{~ms}$, $74.7 \mathrm{~ms}$ and $56.2 \mathrm{~ms}$ in control, short and long term microgravity conditions, bi-directional excitation wave propagation failed to occur as the premature stimulus (S2) did not give the 1D tissue enough time to recover from inactivation (figure 4A). But when the stimulus applied within the vulnerable window, a unidirectional excitation block occurred as shown in figure 4B. It is worth noting that this unidirectional conduction block is in the direction of endocardial section, which is mainly due to the comparatively longer APD in endocardial cell than in epicardial cell. With the increasing of $\Delta \mathrm{t}$, there is enough time for endocardial and epicardial cells in the tissue to recover, resulting in bi-directional excitation conduction in the tissue (figure 4B). The width of the vulnerable time 
window is computed equal to $1.8 \mathrm{~ms}$ and $1.3 \mathrm{~ms}$ in the short and long term microgravity conditions, which are smaller than that of $1.9 \mathrm{~ms}$ in the control condition (figure 4C). As unidirectional block is an essential factor to induce re-entry in $2 \mathrm{D}$ tissue, the decreased vulnerable time window indicated a reduced risk of the occurrence of re-entry in microgravity condition. However, microgravity induced APD reduction, meaning the shortened wavelength of excitation, further facilitated the maintenance of re-entry as observed in previous studies [6].

\section{Discussion and conclusion}

In this study, we performed a series of simulations based on biophysical detailed mathematical models of endocardial and epicardial ventricular myocytes as well as 1D ventricular tissue, evaluating the short and long term microgravity effects on $\mathrm{APD}_{90}$, transmural gradient of $\mathrm{APD}_{90}$ in endocardial and epicardial cells, excitation conduction across the 1D ventricular strand and the vulnerability of the $1 \mathrm{D}$ tissue to unidirectional conduction block. The main findings are that: (I) the reduced $I_{\mathrm{CaL}}$ and increased $I_{\mathrm{NaK}}$ in microgravity conditions resulted in reductions of $\mathrm{APD}_{90}$ and transmural time gradient of $\mathrm{APD}_{90}$, especially in the long time microgravity condition; (II) decreased $I_{\mathrm{CaL}}$ played a main role in decreasing $\mathrm{APD}_{90}$ in microgravity conditions as a whole; (III) the upregulated expression of connexin 43 mildly increased excitation wave conduction velocity in the 1D tissue; (IV) microgravity induced a reduced dispersion of ventricular effective repolarization, that further reduced vulnerability of the $1 \mathrm{D}$ ventricle tissue to generate unidirectional block, especially in the long time microgravity condition.

This study also has some limitations, including: (I) the limitations of the ventricular models that have been described in previous study [5] ; (II) besides ion channel changes, rat tail suspension mimicked microgravity may also induce other changes in heart rate [7] as observed in head down bed rest experiments and the mass alteration of left ventricle [8] in space flight astronauts. Therefore, further studies need to consider these effects on excitation generation and conduction in ventricle tissue. (III) In this simulation study, excitation wave propagation in 1D ventricle strand was simulated, but the substrate property of re-entry in 2D and 3D ventricle tissue is still unclear, further studies need to focus on these issues.

\section{Acknowledgements}

This study was supported by grants from NSFC (61179009, 61601143, 81770328), Shenzhen Science and Technology Innovation Committee (JCYJ20151029173 639477; JSGG 2016 0229125049615).

\section{References}

[1] Yue ZJ, Xu PT, Jiao B, Chang H, Song Z, Xie MJ, Yu ZB. Nitric oxide protects 1-type calcium channel of cardiomyocyte during long-term isoproterenol stimulation in tail-suspended rats. BioMed research international. 2015;2015:780814

[2] Cui Y, Zhang SM, Zhang QY, Fan R, Li J, Guo HT, Bi H, Wang YM, Hu YZ, Zheng QJ, Gu CH, Yu SQ, Yi DH, Li ZC, Pei JM. Modulation of intracellular calcium transient in response to beta-adrenoceptor stimulation in the hearts of 4wk-old rats during simulated weightlessness. Journal of applied physiology. 2010;108:838-844

[3] Zheng H. Effects of simulated microgravity on 3d cultured myocardial cells encapsulated alginate. [D] Harbin, Harbin Institute of Technology. 2012:77-91

[4] Adeniran I, Whittaker DG, El Harchi A, Hancox JC, Zhang $\mathrm{H}$. In silico investigation of a kcnq1 mutation associated with short qt syndrome. Scientific reports. 2017;7:8469

[5] Pandit SV, Clark RB, Giles WR, Demir SS. A mathematical model of action potential heterogeneity in adult rat left ventricular myocytes. Biophysical journal. 2001;81:3029-3051

[6] Zhang H, Kharche S, Holden AV, Hancox JC. Repolarisation and vulnerability to re-entry in the human heart with short qt syndrome arising from kenq1 mutation-a simulation study. Progress in biophysics and molecular biology. 2008;96:112-131

[7] Liang XD, Zhang L, Shen HJ, Chen XY, Wan YF, Li LY, Liang YY, Yu XY, Guo YM, Yu JL, Shu WS, Tan C, Lv K, Xiao Y, Chen XP, Chen SG, Guo JH. Effects of a 45-day head-down bed rest on the diurnal rhythms of activity, sleep, and heart rate. Biol Rhythm Res. 2014;45:591-601

[8] Westby CM, Martin DS, Lee SM, Stenger MB, Platts SH. Left ventricular remodeling during and after 60 days of sedentary head-down bed rest. Journal of applied physiology. 2016;120:956-964

Address for correspondence.

Henggui Zhang

Mailbox 332, Harbin Institute of Technology

Harbin 150001, China

H.Zhang-3@manchester.ac.uk 